\title{
Spectral-based Shadow Detection for Single Image
}

\author{
Xiaoyan $\mathrm{Xu}$ and Xiaoming Liu \\ College of Information Science and Technology,Dalian Maritime University, \\ Dalian 116026, China \\ xxyhlj@126.com
}

\begin{abstract}
Shadow detection for single image is difficult but has wide applications. This paper proposes a novel and fast shadow detection method based on the Tricolor Attenuation Model [1]. In this study, we analyze the spectral property of outdoor light sources to estimate the parameters of TAM. Then our shadow detection method is proposed by integrate the TAM feature and intensity information. Our method can extract shadows for a single and uncalibrated image without any prior knowledge, even for complex scenes. Experimental results show the effectiveness of the proposed method.
\end{abstract}

Keywords: Shadow detection; Tricolor attenuation model

\section{Introduction}

Shadow detection is useful for many computer vision and image analysis tasks, such as object recognition [2], video surveillance [3], environment construction [4], scene geometry [5], and light source location estimation [6]. Furthermore, shadow detection is the foundation of shadow removal. Hence, shadow detection is always the hot spot of research and with rapidly expanding interests.

Although human's eyes can distinguish shadows from an image easily, it is rather difficult for machine vision, to detect shadows especially from a single outdoor image. Since shadows are a difficult phenomenon to model. Their appearance changes with the appearance of the surface they are cast upon. The most straightforward property of a shadow is that it darkens the surface it is cast on. The following work is to distinguish a shadow from a naturally dark surface, e.g., [7-9].

The problem of color invariance emerged from research in the domain of computational color constancy and its application to color-based object recognition. Some methods, e.g., [10], detect shadows on the basis of the observation that, while luminance decreases in shadows, chromatic information remains approximately unchanged. Salvador et al. [11] employed invariance feature to derive intrinsic images for shadow detection.

Jung et al. [12] and Sun et al. [13] discovered two new models derived from a so-called Retinex principle, and well suited for single image shadow detection. Tian et al. [14] proposed the linear model to describe the proportional relationship between shadow and nonshadow background, and used this model to verify and remove shadows. Lalonde [15] and Wang [16] employed multi futures to train and learn shadows for shadow detection.

Many shadow detection methods focus on detecting moving shadows. Moving object can bring about moving shadow. The problem of separating moving cast shadows from moving objects in image sequences is in fact of particular relevance for wider range of applications. The approach described in [17] aims at detecting and classifying background regions which have been covered or uncovered by a moving cast shadow from one frame of the sequence. The method proposed in [18] is dedicated to moving cast shadow detection in monochromatic video sequences. These methods are difficult to be applied to single outdoor image. For in one image less information could be exploited. 
Despite so many approaches have been proposed, many of them are designed for specific applications. When the application environment changed, the algorithms may fail. In addition, most of the proposed methods focus on moving shadows in image sequence, and few can work in single still image. The method proposed in this paper can work on single image without needing priori information.

The remainder of the paper is organized as follows: in Section 2, we review the Tricolor Attenuation Model (TAM) and analysis its parameters based on the spectral property. In Section 3, we present an algorithm described for shadow detection based on the TAM. In Section 4, we give some experimental results, followed by the conclusion and discussion in Section 5 .

\section{Review the Tricolor Attenuation Model}

As shown in Figure 1. Shadows occur when direct light from a light source is occluded.

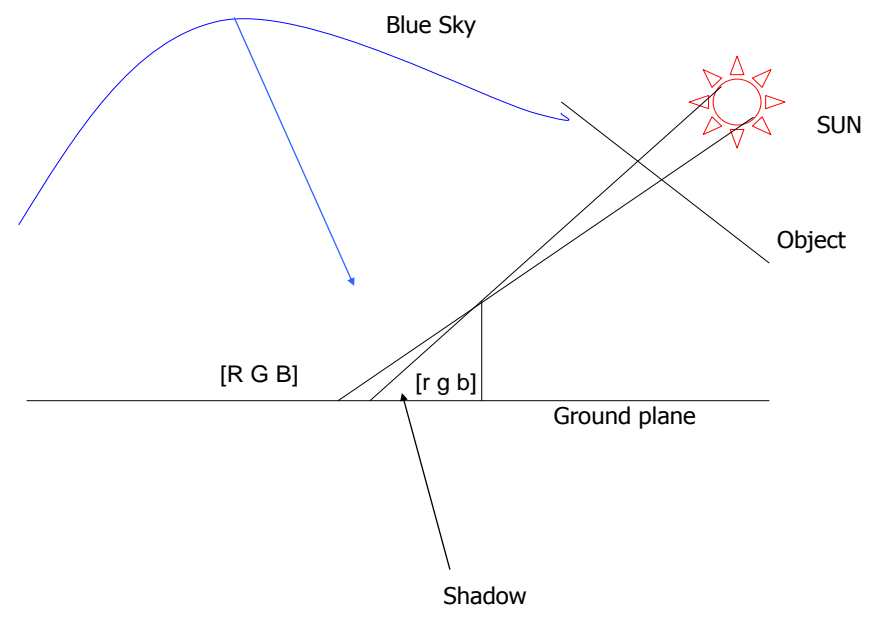

\section{Figure 1. Shadow will Occur when Direct Sunlight is Occluded}

In the non-shadow region, there are two light sources-the sun and the sky whereas in shadow region only the sky light source.

When a non-shadow pixel vector $\left[\begin{array}{lll}F_{R} & F_{G} & F_{B}\end{array}\right]$ on background is occluded by an object from direct sunlight, it will be attenuated by $\left[\begin{array}{ccc}\Delta R & \Delta G & \Delta B\end{array}\right]$ and become a shadow pixel vector $\left[\begin{array}{lll}F_{r} & F_{g} & F_{b}\end{array}\right]$. That is,

$$
\left[\begin{array}{lll}
F_{r} & F_{g} & F_{b}
\end{array}\right]=\left[\begin{array}{lll}
F_{R} & F_{G} & F_{B}
\end{array}\right]-\left[\begin{array}{lll}
\Delta R & \Delta G & \Delta B
\end{array}\right]
$$

Here $\left[\begin{array}{ccc}\Delta R & \Delta G & \Delta B\end{array}\right]$ denotes the tricolor attenuation vector, the relationship among $\Delta R, \Delta G, \Delta B$ is called Tricolor Attenuation Model [1] which can be represented by:

$$
\left[\begin{array}{c}
\Delta R \\
\Delta G \\
\Delta B
\end{array}\right]=\left[\begin{array}{c}
\Delta R / \Delta B \bullet \Delta B \\
\Delta G / \Delta B \bullet \Delta B \\
1 \bullet \Delta B
\end{array}\right]=\left[\begin{array}{c}
m \bullet F_{R} / F_{B} \\
n \bullet F_{G} / F_{B} \\
1
\end{array}\right] \bullet \Delta B
$$

Here, 


$$
\left\{\begin{array}{l}
m=\frac{E_{d a y}\left(\lambda_{B}\right)}{E_{d a y}\left(\lambda_{R}\right)} \bullet \frac{E_{d a y}\left(\lambda_{R}\right)-E_{s k y}\left(\lambda_{R}\right)}{E_{d a y}\left(\lambda_{B}\right)-E_{s k y}\left(\lambda_{B}\right)} \\
n=\frac{E_{d a y}\left(\lambda_{B}\right)}{E_{d a y}\left(\lambda_{G}\right)} \bullet \frac{E_{d a y}\left(\lambda_{G}\right)-E_{s k y}\left(\lambda_{G}\right)}{E_{d a y}\left(\lambda_{B}\right)-E_{s k y}\left(\lambda_{B}\right)}
\end{array}\right.
$$

The illumination on non-shadows is daylight and on shadows (in umbra) is skylight. The SPD of daylight and skylight are denoted as $E_{d a y}, E_{s k y}$ respectively.

\section{1. $m$ and $n$ Estimation}

From formula (3), we can see that $\mathrm{m}$ and $\mathrm{n}$ is depending on $E_{d a y}, E_{s k y}$; therefore, the SPD of daylight and skylight are required.

Some methods assume that outdoor light source is a white one, which is not proper because the spectral of white light (solar irradiance) changed when pass through the out space of the earth. When the light emitted from solar reaches the top of the earth's atmosphere, the atmosphere effect including reflection, scattering, and absorption plays an important role in selectively controlling passage of various wavelength of light. So, the light energy that ultimately reaches the earth's surface is much reduced in intensity as it traverses the atmosphere and the spectral also changed (for example, blue light scatted more, That is the reason why sun looks yellow and sky looks blue).

Sky play an important role in outdoor scene and the shadow generation, although some researcher note that shadow has relation to blue sky (they believe shadow looks more blue) which is not totally true, because the pixel value of shadow also have relation to surface reflection and camera sensor responsibility.

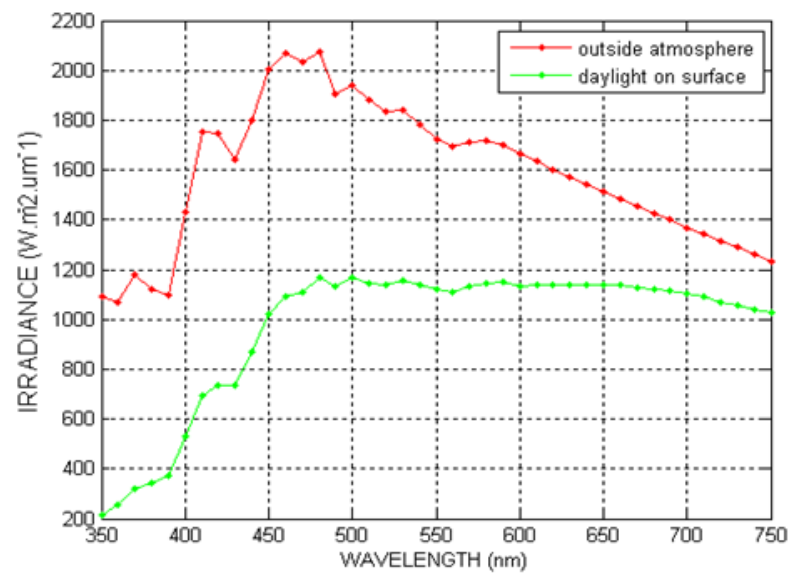

\section{Figure 2. NASA Standard Data of Solar Irradiance Outside Atmosphere and at Earth's Surface [19]; Neighboring Data Points have been Connected by Straight Lines for Illustrative Purpose Only}

In Figure 2, the red line is solar irradiance outside atmosphere. When passing through the atmosphere, the visible light $(380 \mathrm{~nm}-760 \mathrm{~nm})$ is scattered by particles. As shown by the green line, both of the energy and the spectral of the light that ultimately reaches earth's surface (daylight) has been largely changed.

Define $I$ as the incident light and $P$ as the scattered light. According to rayleigh scattering: $P \propto I \bullet \lambda^{4}$, the SPD of skylight can be defined as: $k I \lambda^{4}$. In which, $k$ is a scale factor. Shadows most happen in clear sky weather. In this situation, the proportion of skylight to daylight is about 1/4 [20]. Denote the SPD of daylight as $D$, and the SPD of skylight can be derived from eq. 4 . 


$$
\int_{\Omega} k P \lambda^{-4} d=1 / 4 \int_{\Omega} D d \lambda
$$

The SPD of skylight and daylight are plotted to Figure 3.

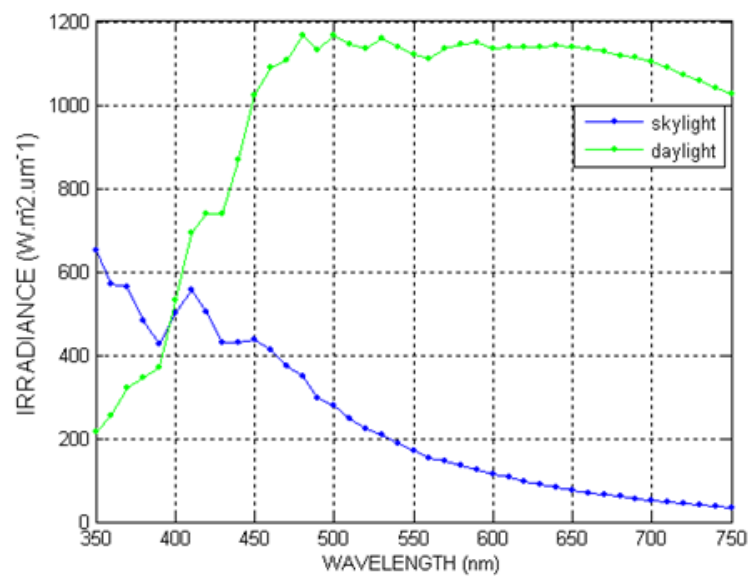

Figure 3. SPD of Daylight and Skylight

In CIE standard, the wavelength at red, green, and blue is $700 \mathrm{~nm}, 546.1 \mathrm{~nm}, 435.8 \mathrm{~nm}$ respectively. The SPD of daylight and skylight from Figure 3 at red, green, blue are tabulated in Table1 (rounding up to the nearest pot).

Table 1. SPD at Red, Green, Blue

\begin{tabular}{cccc}
\hline & Red (700) & Green (550) & Blue (440) \\
\hline daylight & 1102 & 1120 & 867 \\
\hline skylight & 51 & 168 & 430 \\
\hline
\end{tabular}

Substitute this into eq.3.m and $\mathrm{n}$ can be easily calculated.

\section{Shadow Detection Algorithm}

Given a color image $F$, the mean value of $\left[\begin{array}{lll}F_{R} & F_{G} & F_{B}\end{array}\right]$ is calculated by:

$$
\left[\begin{array}{lll}
\overline{F_{R}} & \overline{F_{G}} & \overline{F_{B}}
\end{array}\right]=\frac{1}{M}\left(\sum_{k \in F}^{k=1 \ldots . . M}\left[\begin{array}{lll}
F_{R}^{k} & F_{G}^{k} & F_{B}^{k}
\end{array}\right]\right)
$$

Where $F_{R}^{k}$ denote that the value of $k$ th pixel of $F$ in $R$ channel, and $M$ is the number of pixels in $F$.

Firstly, we calculate $\left[m \bullet \overline{F_{R}} / \overline{F_{B}} n \bullet \overline{F_{G}} / \overline{F_{B}} 1\right]$, and use the maximum channel to subtract the minimum channel. Namely, if $m \bullet \overline{F_{R}} / \overline{F_{B}}>n \bullet \overline{F_{G}} / \frac{}{F_{B}}>1$, the subtractive image $X$ could be obtained by $X=F_{R}-F_{B}$, and vise versa.

In order to improve the robustness of our method, secondly, the subtractive image is combined with grayscale image to form an integrated image $Y$ by:

$$
Y=X \square \operatorname{gray}(F)
$$

Where $\operatorname{gray}(F)$ denotes the grayscale image of color image $F$ and $\square$ denotes pixelwise product. A combination of spectral and intensity properties of imaged shadows is exploited to overcome the limitations of separate methods. 
Finally, the shadow results are obtained by: $\mathrm{S}=\{(x, y) \mid Y(x, y)<T\}$, Where $T$ is the threshold determined by the OTSU method [21].

\section{Experimental Results}

Our method has good results when used on outdoor scenes as shown in Figure 2. All shadows can be detected out. The complex texture in some images does not affect our algorithm response.

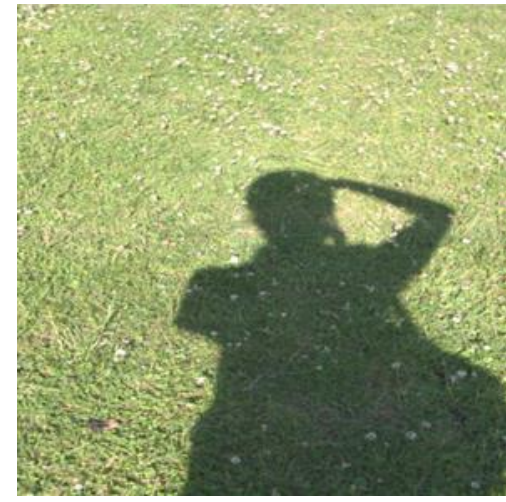

(a)

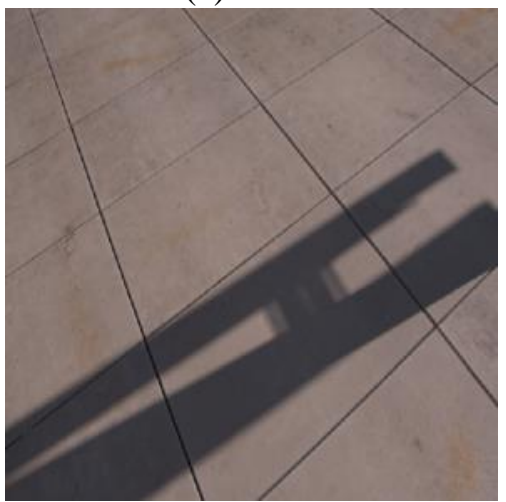

(c)

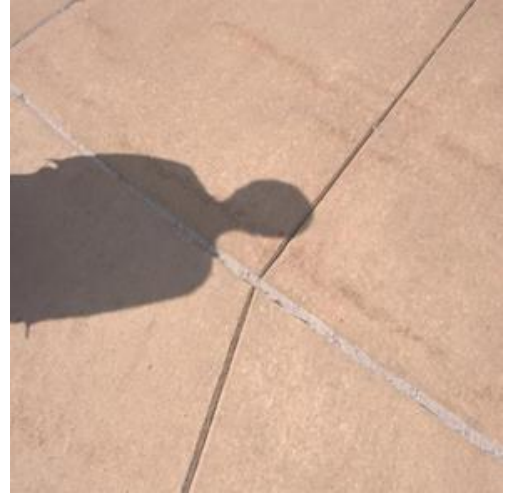

(e)

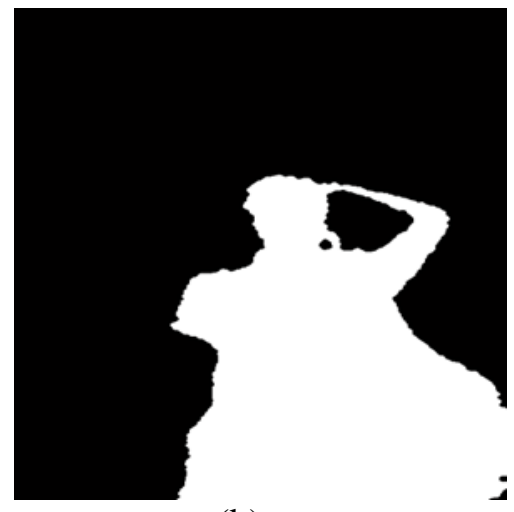

(b)

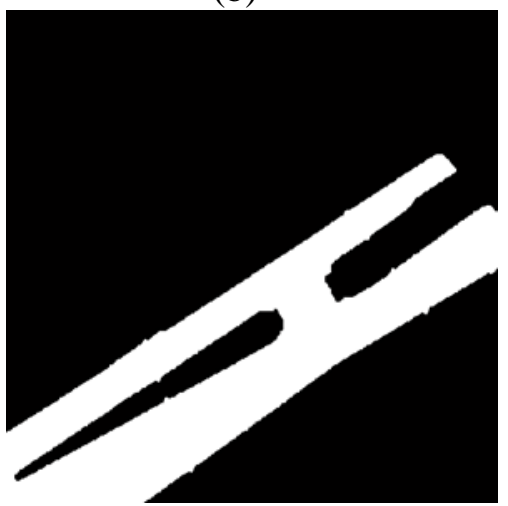

(d)

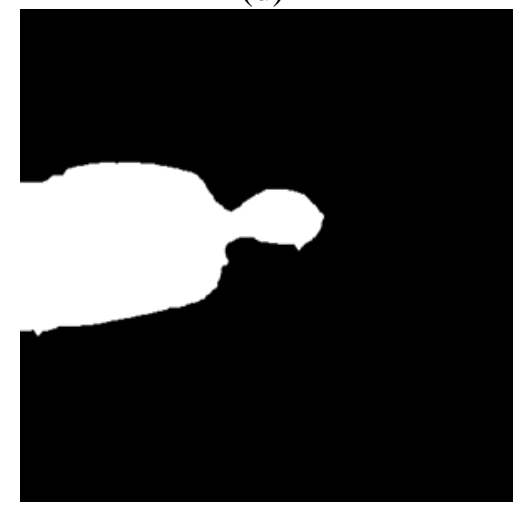

(f) 


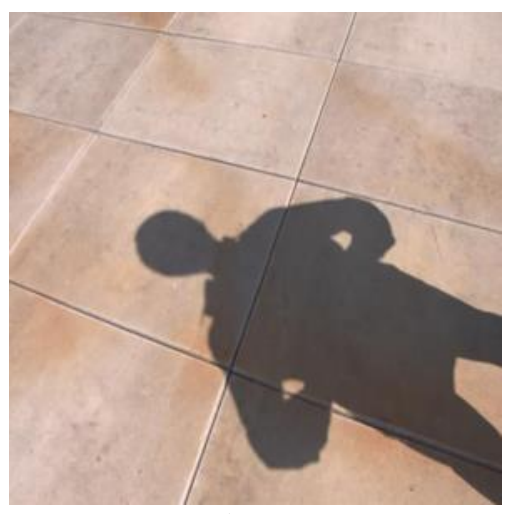

(k)

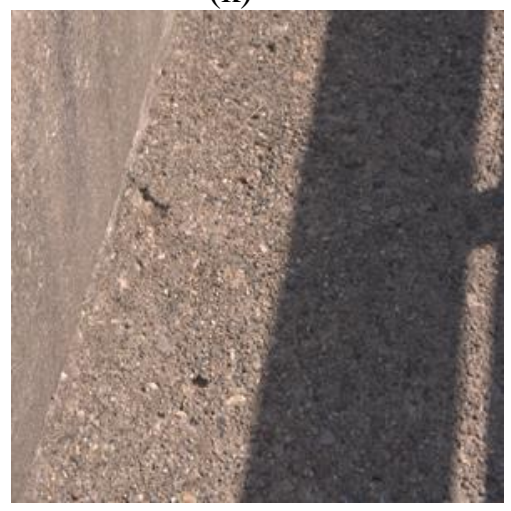

(m)

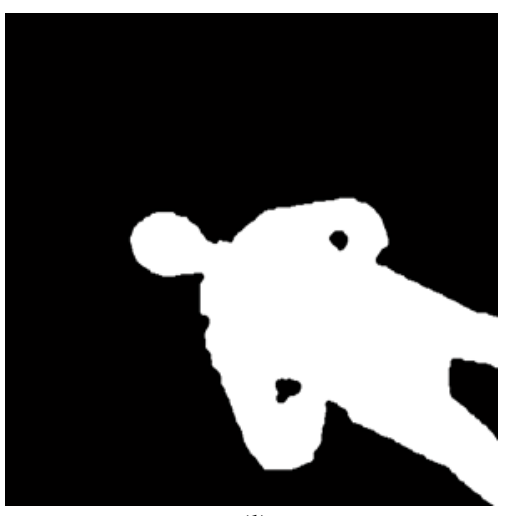

(1)

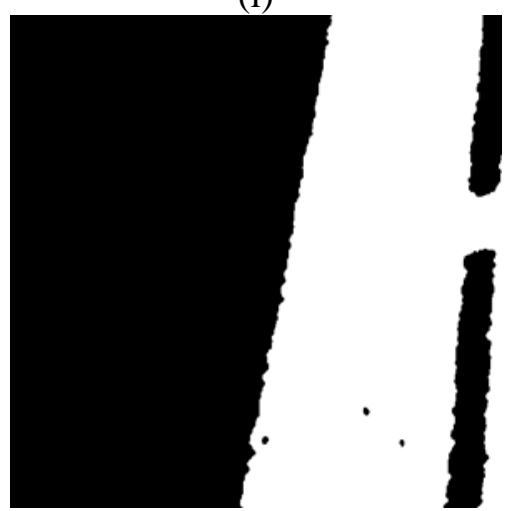

(n)

Figure. 4 Results of our Shadow Detection Algorithm

(a), (c), (e), (k), (m): Original Images

(b), (d), (f), (l), (n): Shadow Images

\section{Conclusion}

Shadow is the common phenomena in nature scenes. For many algorithms in computer vision, dealing with shadows is necessary but a challenging task.

Unlike most previous method using image sequence, our method can extract shadows in only a single and uncelebrated image that takes advantage of the spectral property. We can use only a single color image when detect the shadow in it without needing any assumption (e.g., illumination conditions, scene geometry, camera characteristics or user intervention).

\section{Acknowledgements}

This paper is supported by both National Basic Research Program of China - 973 (No. 2007CB310606) and Fundamental Research Funds for the Central Universities of China.

\section{References}

[1] J. Tian, J. Sun and Y. Tang, "Tricolor Attenuation Model for Shadow Detection", IEEE Transactions on Image Processing, vol. 18, no. 10, (2009).

[2] L. Abraham and M. Sasikumar, "Cloud Extraction and Removal in Aerial and Satellite Images", Proceedings of the International Symposium on Intelligent Informatics, (2012) August 4-5; Chennai, India.

[3] B. Wang, Y. Feng, H. Guo and G. Zhang, "An Effective Shadow Detection Approach in Traffic Scenes", Proceedings of the 30th Chinese Control Conference, (2011) July 22-24; Yantai, China.

[4] S. Kim, C. Coffin, and T. Höllerer, "Robust Relocalization and Its Evaluation for Online Environment Map Construction", IEEE Transactions on Visualization and Computer Graphics, vol. 17, no. 7, (2011).

[5] J.-F. Lalonde, A. A. Efros, and S. G. Narasimhan, "Estimating the Natural Illumination Conditions from a Single Outdoor Image", Proceedings of the IEEE 12th International Conference on Computer Vision, (2009) September 29-October 2; Kyoto, Japan. 
[6] J.-M. PINEL and H. NICOLAS, "Shadows Analysis and Synthesis in Natural Video Sequences", Proceedings of the IEEE Internationa Conference on Image Processing, (2002) September 22-25; NY, United states.

[7] K.-L. Chung, Y.-r. Lin, Y.-H. Huang, "Efficient Shadow Detection of Color Aerial Images Based on Successive Thresholding Scheme", IEEE Transactions on Geoscience and Remote Sensing, vol. 47, no. 2, (2009).

[8] W. Zhang, X. Z. Fang, X. K. Yang and Q. M. J. Wu, "Moving Cast Shadows Detection Using Ratio Edge", IEEE Transactions on Multimedia, vol. 9, no. 6, (2007).

[9] J. Tian, L. Zhu and Y. Tang, "Outdoor Shadow Detection by Combining Tricolor Attenuation and Intensity", EURASIP Journal on Advances in Signal Processing, vol. 1, (2012).

[10] R. Cucchiara, C. Grana, M. Piccardi and A. Prati, "Detecting Moving Objects, Ghosts, and Shadows in Video Streams", IEEE Transactions on Pattern Analysis and Machine Intelligence, vol. 25, no. 10, (2003).

[11] E. Salvador, A. Cavallaro and T. Ebrahimi, "Cast Shadow Segmentation Using Invariant Color Features", Computer Vision and Image Understanding, vol. 95, no.2, (2004).

[12] J. Chanho, K. Wonjun and K. Changick, "Detecting Shadows from a Single Image”, Optics Letters, vol. 36, no. 22, (2011).

[13] J. Sun, J. Tian, Y. Du and Y. Tang, "Retinex Theory-based Shadow Detection and Removal in Single Outdoor Image", Industrial Robot, vol. 36, no. 3, (2009).

[14] J. Tian and Y. Tang, "Linearity of Each Channel Pixel Values from a Surface in and Out of Shadows and Its Applications", Proceedings of the IEEE Computer Society Conference on Computer Vision and Pattern Recognition, (2011) June 20-25; CO, United states.

[15] J.-F. Lalonde, A. A. Efros, and S. G. Narasimhan, "Detecting Ground Shadows in Outdoor Consumer Photographs", Proceedings of the 11th European Conference on Computer Vision, (2010) September 5-11; Crete, Greece.

[16] Z. P. Wang, J. D. Tian, Y. D. Tang, Y. Z. Zhang, Y. Xia and L. Wang, "Shadow Detection Using Multifeatures in SVM Classifier", Proceedings of the 2nd International Conference on Precision Mechanical Instruments and Measurement Technology, (2014) May 30-31; Chongqing, China.

[17] J. Stauder, R. Mech, and J. Ostermann, "Detection of Moving Cast Shadows for Object Segmentation", IEEE Transactions on Multimedia, vol. 1, no. 1, (1999).

[18] C. Bräuer-Burchardt, "Detection of Strong Shadows in Monochromatic Video Streams", Proceedings of the 13th Scandinavian Conference on Image Analysis, (2001); Springer Verlag, Berlin.

[19] G. Wyszecki and W. S Stiles, "Color Science: Concepts and Methods Quantitative Data and Formulae", Second Edition, Wiley-Interscience Publishers, Wiley, New York (1982).

[20] J. Boland, L. Scott and M. Luther, "Modelling the Diffuse Fraction of Global Solar Radiation on Horizontal Surface", Environmetrics, vol. 12, no. 2, (2001).

[21] N. Otsu, "A Threshold Selection Method from Gray-level Histograms", IEEE Transactions on Systems, Man, and Cybernetics, vol. 9, no. 1, (1979).

\section{Authors}

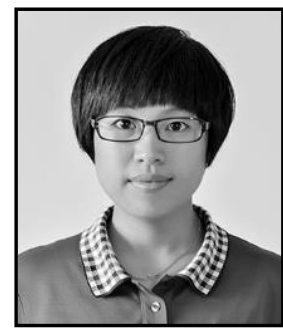

Xiaoyan Xu, She received her M.S. degree in Signal and information processing from HeiLongjiang University, China, in 2008. She is now a $\mathrm{Ph} . \mathrm{D}$. candidate in College of Information Science and Technology, Dalian Maritime University. Her main research interests in image processing and pattern recognition.

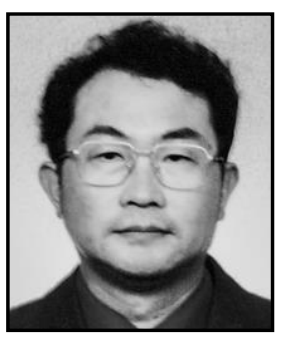

Xiaoming Liu, $\mathrm{He}$ received his M.S. and Ph.D. degrees in Communication and information system from Dalian Maritime University, China. He is a professor in College of Information Science and Technology, Dalian Maritime University. His research interests include radar signal processing, electronic information system and image processing. 
International Journal of Hybrid Information Technology Vol.8, No.4 (2015) 